\title{
NESTORE: a Multidomain Virtual Coach for Active and Healthy Ageing
}

\author{
Maria Renata Guarneri ${ }^{1}$, Alfonso Mastropietro ${ }^{2}$, Giovanna Rizzo $^{2}$ \\ ${ }^{1}$ Politecnico di Milano, Milano, Italy \\ ${ }^{2}$ Istituto di Bioimmagini e Fisiologia Molecolare, CNR, Segrate (MI), Italy \\ Mariarenata.guarneri@polimi.it
}

\begin{abstract}
Technology can play a key role in support of the needs of the ageing population. In this direction, the rapid development of the ICT, and in particular mobile technologies, offers an important opportunity to address the development of an integrated solution to support active and healthy ageing. Whilst technology can potentially have a significant impact on health and wellbeing, to date uptake of digital health technologies has been problematic in a number of wide-scale studies. Literature has cited confidence, the stigmatizing aesthetics of products, meaningfulness of technology in the broader context of the persons' life, ease of use and integration into everyday routines as important factors of non-acceptance. With the aim of overcoming the above limitation, we have gathered a multi-disciplinary consortium to develop an integrated solution that, strongly leveraging user participation and co-design as well as state-of-the-art technologies, offers a virtual coach service to elderly people so that they can maintain wellbeing and independence. The solution, in addition to being multi-technology, has the ambition of addressing wellbeing in a holistic manner taking into consideration several dimensions. NESTORE will start in September/October 2017 and will last three years. NESTORE involves 16 partners from 7 European countries. The paper presents the approach to the research and the envisaged results.
\end{abstract}

Keywords: Healthy ageing, wellbeing, smart objects, interaction design, user empowerment, self-management

\section{Introduction}

The NESTORE (Non-intrusive Empowering Solutions and Technologies for Older people to Retain Everyday life activity) project has been recently funded by the H2020 Programme under Strategic Challenge 1 "Health, demographic change and wellbeing" in response to the call on healthy ageing.

The aim of the project is to develop a companion - NESTORE - that, as the mythological Nestor, can give advice to older people so that they can maintain their wellbeing and their independence at home, based on experience and on 
understanding the current situation. The experience of our modern NESTORE is based on well-grounded psychological and behavioural theories jointly with relevant know-how on the ageing process, while the current situation is understood on the basis of a comprehensive sensors' system able to monitor the different key parameters. An intelligent system, based on a cloud and leveraging Decision Support logics, will deliver "advice and coaching", which will be offered via the companion, embodied in a smartphone or an intelligent tangible object, according to the user's preferences and interests.

The core idea of NESTORE is linked to the design of pathways that, according to user preferences and inclinations, leverage existing personal capabilities to support healthy lifestyles and overall wellbeing.

The paper presents first an overview of the wellbeing dimensions covered by NESTORE; the core of the paper focuses on the key concept and on the approach to be followed in the development of the solution. An overview of the validation approach will then be presented.

\section{The five key dimensions of wellbeing}

Ageing is a multi-dimensional and multi-directional process. It involves the social, economic, physical, psychological and cognitive spheres and all these characteristics of a person and its context is strongly interconnected. Crucial factors that affect the wellbeing and along which the solution proposed by NESTORE will be developed are: i) physiological status and physical activity behaviour, ii) nutrition, iii) cognitive capabilities, iv) mental and psychological wellbeing, and v) social interaction.

Physiological status and physical activity behaviour. Ageing is characterized by motor function impairment such as coordination difficulty, slowing of movements, and difficulties with balance and gait [1]. These deficits may have a negative impact on the ability to perform daily activities and may result in a reduced independence and self-confidence, thus increasing the risks of traumatic events (i.e., falls) and social exclusion. Progressive and generalized loss of skeletal muscle mass and strength (i.e., sarcopenia) is physiologically associated with ageing and has an important impact on wellbeing [2]. Physical activity is a most effective intervention to counteract skeletal muscle impairment [3]. The aerobic and anaerobic physical activities should be tailored to the older people in order to retain or improve their cardiovascular fitness, muscle strength and overall balance.

Nutrition. The process of ageing involves a steady decay of the metabolic flexibility [4]. The whole metabolism is more sensitive to unbalanced diets and to suboptimal nutrition. Ageing also involves the senescence of tissues and metabolic processes, which results in altered absorption of nutrients together with decreased capacity in detoxifying by-products. Therefore, during this period of life, a balanced diet together with optimal dietary patterns is key in order to maintain the whole body homeostasis and to overcome the naturally occurring physiological limitations of the subject. 
Cognitive capabilities. Ageing is also related to cognitive decline, one of the most worrying age-related changes in later adulthood [5]. Whereas more knowledge-driven domains tend to be maintained relatively well, the more biologically-driven fluid abilities show average decline throughout adulthood, with accelerated decline late in life and prior to death. Cognitive training interventions are known to be successful in improving the trained abilities, with little evidence for transfer to unrelated areas or real life [6] The trainings, to be successful, need to engage older adults in novel tasks that are ecologically valid and easily integrated into the daily life routines.

Mental and psychological wellbeing. Other important aspects are the preservation of mental wellbeing of older people [7]. In order to ensure a mental wellbeing, interventions should focus on psychological factors (participation in activities that are relevant to an individual's personal goals, emotionally close personal relations, protection of a positive sense of self through prevention of discrimination), socioeconomic factors (i.e., financial security to meet one's personal goals), and physical health (i.e., successful management of physical conditions to ensure high subjective health).

Social wellbeing. Strictly related to the previous dimension, another important aspect is the preservation of the social capital provided by older people. The socio-economic status, social integration and high personal competencies are related to higher subjective wellbeing [8]. The quality of the social contacts and the availability of emotionally meaningful social relations is, as well, an important factor to consider for a better wellbeing. There is evidence that voluntary work, as well as the educational and social activity group interventions, can improve the mental health and prevent social isolation and loneliness among older people.

\section{The NESTORE Concept}

With the objective of addressing the five dimensions described above with an integrated holistic approach, the solution will be developed in co-design with the users and will the potential of ICT to meet their needs. Co-design will ensure that users can be emotionally involved, thus achieving a significant step ahead in the empowerment of citizens and in promoting and actively sustaining active ageing.

\subsection{The NESTORE Vision}

The vision proposed is that of NESTORE as a friend and a companion. As a trusted friend, NESTORE can also be accepted as a coach.

NESTORE knows me and understands my emotions. We have only recently met, but it is as if we have known each other for our entire life. It is really an empathic and emotional relation that has been developed. I know you can count on him for the right support, for that one hint of encouragement when my will is not strong. Now it is really much more fun to take a walk, to perform those boring exercises needed to keep in good shape; thanks to NESTORE my social life is still active and I have even met new friends. Occasionally we play together and that also exercises my brain. He 
is there when I need him, but he also understands when I want to be left on my own. NESTORE really is the "answer" to my needs.

NESTORE is capable of valorising the capabilities of the person and helps him/her to select the vocational path along the five dimensions of the well-being profile, i.e. nutrition, physical activity, cognitive, social and mental. NESTORE is also aware of the family ties and leverages them to add a human touch to the technology based service offering.

\subsection{The NESTORE Services}

This section provides an overview of the potential services NESTORE can provide to the target audience, and what is meant by "pathways of wellbeing". NESTORE is designed to address the needs of the older people of tomorrow, therefore the solution must be flexible to include technologies yet to come, and it must offer open and standardised interfaces so that new services - meeting new needs and preferences can be offered by third parties. Computer literacy and capabilities to use technologies will also increase in the future. To better understand the users, the consortium will conduct focus groups, not only with people that today are in target (i.e. over 65) but also with people that today are in their 50ies, to understand how they see their future and what would be on target in 15 years and more from the present time.

Typical users of NESTORE are people that are familiar with modern technologies, are in a reasonably good health, have recently retired or about to retire and have a social life they want to keep. They remain users of NESTORE throughout the process of ageing. With age, NESTORE knows the person, becomes aware of possible degradation of functional capabilities and provides suggestions and services to compensate such shortcomings.

To start the initial definition of services, NESTORE works on the assumption that older people recently retired want or need to find new interests: new life, new challenges! They also have finally time to cultivate better existing interests. The theoretical base for the design of the solution starts from the Selection, Optimization and Compensation (SOC) model [9] that outlines general-purpose processes of specialization by selection of developmental pathways, preferences or goals. According to SOC, the goal of NESTORE is to empower the latent reserve capacity of older people to limit the age-related loss of skills, in relation to the 5 dimensions addressed by NESTORE. To foster motivation, NESTORE helps the user to select and identify a final purpose "the wellbeing pathway" (based on the risk perception, self-efficacy and outcome expectancies); it then facilitates the action planning and the maintenance of self-efficacy through the provision of a set of actions aimed at the achievement of the goal. Finally, NESTORE monitors the user's progresses in order to propose recovery actions in case of impediments or actual loss of motivation.

Adopting SOC, NESTORE will define specific pathways that meet user preferences and whose aim is to allow reaching, by the older people, the maximum capacity of their latent reserve, that guarantees an active and healthy ageing. Such definition is related to the Selection component of the SOC model. It will then identify the building blocks that specify each pathway; this is related to the 
acquisition, application and integration of resources involved in attaining a higher level of functionality (internal or external) in all the dimensions (Optimization). Such building blocks can be represented as possible actions that the user shall perform to pursue its pathway and that are suggested, monitored and provided by the Virtual Coach. Finally, NESTORE monitors the user's level of motivation and engagement and intervenes to restore and manage impediment or loss of motivation (Compensation).

Following the approach described above, the following example of service provision scenario can be devised:

John is 65 and has recently retired. He loves gardening and now that he is free, he would like to spend more time in his garden. Mary, his wife, has in mind some renovations that have been postponed due to lack of time, bad weather, etc. In addition, doing some exercise in the garden will help to keep fit.

Gardening is the topic of the coaching activity selected by John. NESTORE has learned about the interest in gardening on the basis of John's profile and John has confirmed this. By means of the profile data and other data coming from observing the user, NESTORE sets quantitative goals that optimize John's health and wellbeing status according to the NESTORE Integrated Wellbeing Model and proposes a set of activities that foster the achievement of the defined goals. NESTORE then monitors John's progresses in order to outline and manage the possible loss of motivation. The services proposed by NESTORE come from a combination of required activities according to the Integrated Wellbeing Model, i.e. the need to perform certain physical exercises to keep muscle strength or to maintain the cardio-respiratory function, to ensure a certain level of social activities, etc.; these services will support the user in performing them within the topic of gardening, making them more pleasurable for the user. As John performs the activities proposed by NESTORE, he can gain points on the NESTORE system that will reward him with new services, such as e.g. discounts. Indeed, offering open interfaces to third parties, service providers may join the NESTORE network, so that they can advertise their services directly on the platform, and join the stakeholders' network. Within NESTORE we will develop a limited number of potential pathways, based on the results of the focus groups. Such pathways will be demonstrated and tested in the pilots.

\subsection{The NESTORE Technologies}

In order to achieve such an ambitious vision, the research brings together different technologies and resources from several European partners and leverages the most advanced state of the art technologies. With a bottom-up approach, the following areas of research are addressed:

- Information gathering technologies, to learn things about the user. Here we include Sensing Technologies with a wide approach, including passive and active monitoring, based on a pervasive IoT. We aim at knowing the user "intimately" therefore we want to understand habits, feelings and emotions, the environment, the social life and other key information about the user, including - of course - the health status. According to a human model that covers the five dimensions of the NESTORE interventions, we will investigate 
several conventional and unconventional sensing technologies that can generate data about the user achieving advanced personalisation of the solutions and related service offerings. Such technologies include wearable sensors, cameras, environmental sensors, social networks, games, etc. The actual selection will be guided by the results of focus groups with users.

- Technologies for information analysis to understand the user and to generate the appropriate feedback. NESTORE will support the development of pathways of wellbeing, proposing different actions and services and then coaching the user so that motivation can be sustained in time. The data collected are analysed by a decision support system (DDS) that determines the type of intervention. The NESTORE DSS will be based on a three-layer structure: 1) a short-term analysis that analyses data on a daily basis; 2) a long-term analysis that looks at trends and is able to detect change and adapts the coach in the long term, following the changing needs of people as they age; 3 ) a combined short and long-term analysis to provide personalized plans as part of the Coaching function.

- Technologies to convey the feedback to the users, i.e. the Coaching System, the embodiment of NESTORE. From the point of view of the user interaction NESTORE combines different modalities (tangible voice and touch) and different devices (smartphones, tablets, PCs, smart TV, etc.). Within the project we will investigate the use of tangible objects that may favour an affective interaction, supporting the user in a continued use of the system. Gamification mechanics will be used to support motivation, rewarding the users for positive behaviours.

- Technologies to deploy and control sensing environments: Pilot Sites. All the technologies used and developed will be integrated to offer an intelligent system easy to configure, deploy and replicate in various pilot sites. Existing IoT platforms and cloud infrastructures represent today a viable solution to implement features envisioned by Smart Environments concept. Cloud services will be beneficial for two reasons: 1) there are fewer components deployed at home/site. 2) A unified view is offered for geographically distant sites. The management and configuration of the pilot site are simplified because less local interventions are required. The intelligence of the system is deployed in the cloud and the Control Centre interacts with the cloud to transparently tune or upgrade the system.

\section{The NESTORE Methodology}

\subsection{Co-design and Participatory Approach}

The methodological approach followed in NESTORE provides for users to be involved in the design of the solution throughout the project duration. The methodology adopted draws on the value of 'thinking with things' as a means to build an understanding of the factors end-users identify as being important in the design of digital health services and devices.

Literature highlights that the reason for non-acceptance of health technologies is complex. The role of the design is to better understand the broader physical and social environments in which services and technologies will operate and how they relate to the contexts of the end users' lives. NESTORE will adopt co-design tools and methods that will seek to engage and elicit information related to perception, acceptance and usability of technology to support healthcare. Such methods have been shown to be beneficial in the evaluation and design of health-care interventions [10]. 


\subsection{Development of the Solution}

Development of a multi-domain unobtrusive monitoring system, to monitor physiological and behavioural data related the five domains of wellbeing. Technical solutions based on advanced non-invasive monitoring systems, such as wearable and environmental sensors (indoor/outdoor), multifunction tangible objects, serious games, will be optimized and integrated. The Wearable monitoring system includes sensors suitable for end-user self-operating and allowing for an accurate assessment of meaningful physiological parameters. The Environmental monitoring system includes sensors for indirect monitoring of behavioural information, related for instance to nutrition, daily living activities patterns, physical activity, etc. Multifunction tangible objects include sensors suitable for indirect recognition of emotional and mental status. The Serious games integrate sensors suitable for monitoring cognitive abilities, such as memory performance or verbal fluency.

Development of an intelligent and innovative ICT Decision Support System (DSS), able to analyse the seniors' behaviour, tracking changes and compliance to active ageing guidelines, providing personalized target behaviours towards the adoption and maintenance of healthy lifestyle. For such a system to work effectively as a DSS, person's goals, overall cognitive/physical/mental and social status need to be assessed together with a profile of a person's daily life activities monitored using technology-based tracking systems in order to provide a reference frame and basis for the DSS that includes an individualized real-life approach rather than a mere population-based approach based on maximum performance laboratory-based assessments.

Development of an active coaching system, which, based on the user profile and needs, stimulates and engages older people with personalized coaching activities in a single or multiple wellbeing domains, following the Selection, Optimization and Compensation (SOC) model [9]. The coach will provide the necessary information to appropriately support the different phases of the behaviour change intervention, following the health action process approach (HAPA) [11]. The coach will be developed as a conversational agent able to assume different forms. In particular, it can be embodied in a physical companion that aims at establishing a trustful and affective communication with the user through multimodal communication channels. The emotionality of the communication can be supported by affective interactions with the coach, both through physical interactions with the tangible embodiment of the coach and through the semantic analysis of the conversations between the user and the coach.

System validation. The solution will be investigated with the target user communities in three EU locations (Spain, Italy and The Netherlands) in order to take into account differences concerning nutrition habits and lifestyles as well as the cultural and social environment. The system will be tested in terms of usability and ease of user interaction, acceptability and effectiveness.

\subsection{Validation of Solution through Pilots in Real-life Settings}

Because of their different social organisation, end-user's recruitment will be based on participation of different entities and networks of each piloting sites, such as: older 
people associations, senior networks and volunteers' associations, primary care centres, and general practitioners, neighbourhood nurses, third age universities, social care providers, seniors' centres.

Different tools will be used in the piloting phase to collect the user's needs and preferences that the resulting ICT platform should take into account. The results of these tools execution will be considered including different functionalities and/or components of the platform. The first user testing will be focused on showing to the user the implemented functionality considering the requirements collected from the questionnaires and focus groups, on finding out if the User interface design (UI) concept was understandable and on verifying if they see the resulting service as something necessary and usable. This testing validated the functionality included in the prototype and allowed a refinement of the requirements collected in the focus groups and questionnaires, involving new functionalities and changes/additions of architecture components.

To evaluate the correctness of the measurements provided by the NESTORE's monitoring system, a measurement validation process will be carried out comparing measures obtained by sensors and devices with standardized measures including the same type of information obtained by standardized and validated tools.

The effectiveness of the coaching activity proposed by NESTORE will be evaluated in a sub-study. We will add some other assessment variables to analyse the impact in each one of the 5 domains included in NESTORE. Some complementary measurements will help to provide information on possible positive effects on the body produced by using the NESTORE platform.

\section{Conclusions}

NESTORE has the ambition to offer a solution that addresses healthy ageing with a wide-reaching and integrated approach, and by testing such solution in real-life settings in order to accelerate the process for digital innovation and to achieve wide acceptance by the target audience. The proposal has also the ambition to contribute to some aspects of the strategy of Triple Win for Europe, with specific attention to the older population.

- Improve the health and quality of life of citizens; by providing a solution along the concept of "wellbeing pathways" supporting senior citizens not only to keep their health but also to improve their quality of life with personalized and meaningful suggestions that encounter their preferences and fulfill self-achievement.

- Support the long-term sustainability and efficiency of health and social care systems; by providing a solution that allows people to live longer in their homes and a system that eases communication with the surrounding social.

- Enhance the competitiveness of EU industry creating economic growth opportunities and jobs in the Silver Economy; NESTORE aims at providing an open solution that allows the provision of services by third parties by means of open API. In this manner novel services can be designed by innovative companies to reach a market that through NESTORE can offer loyalty programs. NESTORE therefore offers growth opportunities to innovative SMEs in the health and social care field. 


\section{Acknowledgments.}

NESTORE is co-funded by the European Commission under the H2020 program, GA769643. The authors wish to thank all the project partners for their contribution to the project.

\section{References}

[1] Nelson ME, Rejeski WJ, Blair SN, et al. Physical activity and public health in older adults: recommendation from the American College of Sports Medicine and the American Heart Association. Med Sci Sports Exerc. 2007;39(8):1435-1445.

[2] Di Tano G, Fulle S, Pietrangelo T, Bellomo R, Fanò G. Sarcopenia: characteristics, genesis, remedies. Sport Sci Health. 2005;1(2):69-74.

[3] Freiberger E, Sieber C, Pfeifer K. Physical activity, exercise, and sarcopenia - future challenges. Wien Med Wochenschr. 2011;161(17-18):416-425.

[4] Calçada D, Vianello D, Giampieri E, Sala C, Castellani G, de Graaf A, Kremer B, van Ommen B, Feskens E, Santoro A, Franceschi C, Bouwman. The role of low-grade inflammation and metabolic flexibility in aging and nutritional modulation thereof: a systems biology approach. Mech Ageing Dev. 2014 Mar-Apr;136-137:138-47.

[5]Harada CN, Natelson Love MC, Triebel K. Normal cognitive aging. Clin Geriatr Med. 2013;29(4):737-752.

[6] Klimova B, Valis M, Kuca K. Cognitive decline in normal aging and its prevention: a review on non-pharmacological lifestyle strategies. Clin Interv Aging. 2017 May 25;12:903910 .

[7] Steptoe A, Deaton A, Stone AA. Psychological wellbeing, health and ageing. Lancet. 2015 Feb 14; 385(9968): 640-648.

[8] Charles S, Carstensen LL. Social and Emotional Aging. Annu Rev Psychol. 2010; 61: 383409.

[9] Freund, A. M., \& Baltes, P. B. (2000). The orchestration of selection, optimization and compensation: An action-theoretical conceptualization of a theory of developmental regulation. In W. J. Perrig \& A. Grob (Eds.), Control of human behavior, mental processes, and consciousness: Essays in honor of the $60^{\text {th }}$ birthday of August Flammer (pp. 35-58). Mahwah, NJ: Lawrence Erlbaum.

[10] Schmittdiel, J. A., Grumbach, K., \& Selby, J. V. (2010). System-based participatory research in health care: An approach for sustainable translational research and quality improvement. Annals of Family Medicine, 2010 May-Jun;8(3):256-9.

[11] Schwarzer, R. (1992). Self---efficacy in the adoption and maintenance of health behaviors: Theoretical approaches and a new model. In R. Schwarzer (Ed.), Self-efficacy Thought control of action (pp. 217-242). Washington, DC: Hemisphere. 